\title{
Cost Functions for Assessment of Vehicle Dynamics
}

\author{
Dzmitry Savitski \\ Automotive Engineering Department \\ Ilmenau University of Technology \\ Ilmenau, Germany \\ dzmitry.savitski@tu-ilmenau.de \\ Pavel Nedoma, Jaroslav Machan \\ Skoda Auto \\ Mlada Boleslav, Czech Republic \\ pavel.nedoma@skoda-auto.cz
}

\author{
Jiri Plihal \\ Institute of Information Theory and Automation \\ Academy of Sciences of the Czech Republic \\ Prague, Czech Republic \\ j.plihal@volny.cz
}

\author{
Valentin Ivanov, Klaus Augsburg \\ Automotive Engineering Department \\ Ilmenau University of Technology \\ Ilmenau, Germany \\ valentin.ivanov@tu-ilmenau.de
}

\begin{abstract}
The paper addresses methodology of the assessment of vehicle dynamics on the basis of a set of cost functions. The proposed concept covers different domains like longitudinal, lateral and vertical dynamics with a possibility to deduce a complex cost function having three main attributes: (i) dimensionless form, (ii) range from 0 to 1 , and (iii) weighting factors for each individual domain. The attributes (i) and (ii) are subjected to the comparison of baseline, reference, and actual values of corresponding parameters of vehicle dynamics like acceleration, yaw rate, slip and others. The attribute (iii) links to the choice of weighting factors from the analysis of vehicle maneuvers. Application fields of the developed individual and complex cost functions are assessment of vehicle performance and stability, optimization of relevant control systems, and choice of proper control strategies / tuning of control gains by autonomous or cooperative operation of several systems of vehicle dynamics control. The paper introduces the implementation of the proposed methodology by the example of Electronic Stability Program (ESP) system. Case studies illustrate results of the calculation of the cost functions for the avoidance and slalom maneuvers. The corresponding computational procedures are based on full vehicle software simulator, validated with experiments on the real vehicle.
\end{abstract}

Keywords-vehicle dynamics; cost function; weighting factors; electronic stability control; modeling

\section{INTRODUCTION}

Recent developments in automotive control raise a number of issues that address the complex assessment of vehicle dynamics for the system benchmarking and the optimization of control algorithms. The reason is that a typical state-of-the-art ground vehicle belongs to the class of multi-actuated objects. Such a vehicle involves different subsystems, which have functions of dynamics control and can operate both autonomously and in integration with one another. For instance, the longitudinal and lateral vehicle dynamics can be simultaneously controlled through anti-lock braking (ABS), traction slip control, electronic stability program (ESP), active differential, torque vectoring, and so on. The resulting integration of subsystems calls for the development of an

The research leading to these results has received funding from the European Union Seventh Framework Program FP7/2007-2013 under grant agreement no. 284708 . appropriate analytical method that can assess the performance of the controlled vehicle maneuvers from viewpoint of longitudinal, lateral and vertical dynamics, driver comfort, energy efficiency and other factors. The reasonable solution for such a method can be the implementation of a set of cost functions taking into account vehicle maneuver conditions, road parameters, and operational characteristics of the vehicle.

The compilation of cost functions can use conventional indicators of vehicle stability, efficiency, comfort and so on. The relevant vehicle dynamic parameters are adequately described in various state-of-the-art reference books [1-3]. Within this context, an appropriate solution can include the application of normalized parameters that is of special interest by the assessment of combined lateral and longitudinal maneuvers. Examples of normalized and dimensionless description of tire forces and moments, camber and slip angles, and wheel slip ratios were proposed in works $[4,5]$. Other studies are also known where more specified methods have been introduced to describe the cost functions. For instance, the work [6] proposes the combined assessment of lateral and ride dynamics using the frequency-dependent weighting index of lateral acceleration.

The cost functions presented in the actual study are based on an original methodology that was firstly introduced in [7]. The methodology uses a set of global functions derived from the typical parameters adopted for the evaluation of vehicle response characteristics, such as yaw rate, acceleration, sideslip angle, and so on. The individual components of the functions are connected through weighting factors, which can be variable depending on the kind of vehicle maneuver as well as on the driving conditions. The details of the methodology are given in the subsequent sections of the paper. The procedure of calculation and the application of cost functions will be illustrated for two case studies covering the complex vehicle dynamics during avoidance and slalom maneuvers.

\section{GENERALIZED CONCEPT OF COST FunCTIONS}

The performance and effectiveness of a vehicle maneuver can be estimated through parameters related to different domains. Domains under discussion are longitudinal, lateral 
and vertical dynamics, driver comfort, handling, agility and so on. As a result, diverse concepts of cost functions can be potentially derived. Within the framework of the presented study, the requirements to the concept in question are proposed as follows:

- Each domain has an individual cost function.

- Arguments of the individual cost functions are based on corresponding parameters that can be either observed by conventional vehicle sensors or estimated from models embedded into on-board processors.

- Weighting factors are assigned to each argument of the individual cost functions. The sum of weighting factors within certain domain is equal to 1 .

- Arguments of the individual cost functions have a numerical form that is normalized to the range $[0 ; 1]$.

- To define a complex cost function for several domains of vehicle dynamics, additional weighting factors have to be assigned for each domain. The sum of weighting factors for all domains analyzed by the complex cost function is equal to 1 .

Fig. 1 illustrates the process of composition of individual and complex cost functions. This process can be described through the following computation sequence.

1) Choice of a set of parameters of vehicle dynamics for each domain. For example, the lateral acceleration, the yaw rate and the side slip angle can be attributed to the domain "Lateral dynamics".

2) Transformation of the parameters to the dimensionless form in the range $0 \ldots 1$. The methodology under discussion proposes the following procedure for normalization (based on root mean square function):

$$
f(x)=\frac{\sqrt{\frac{1}{n}\left(\sum_{i=1}^{n}\left(x_{i}^{r e f}-x_{i}^{a}\right)^{2}\right)}}{\max \left(X^{a}\right)-\min \left(X^{a}\right)},
$$

where $x$ is the parameter (e.g., acceleration); $X(n)$ is the vector of $x$-values for the vehicle maneuver under assessment. The indexes ,,ref" and , $a$ " are designated for the reference and actual values correspondingly. Each parameter of the cost function has an individual procedure of the definition of reference characteristics. It should be pointed out that the function $f(x)$ will be in the range $[0 ; 1]$. The value " 0 " should be considered as "the best case": the actual values of a variable coincide with the reference values. The value "1" should be considered as "the worst case".

3) Individual weighting factors are assigned to each of parameters in accordance with conditions like

$$
\sum_{i=1}^{m} w_{A i}=1 ; \sum_{i=1}^{l} w_{B i}=1 ; \ldots ; \sum_{i=1}^{k} w_{N i}=1
$$

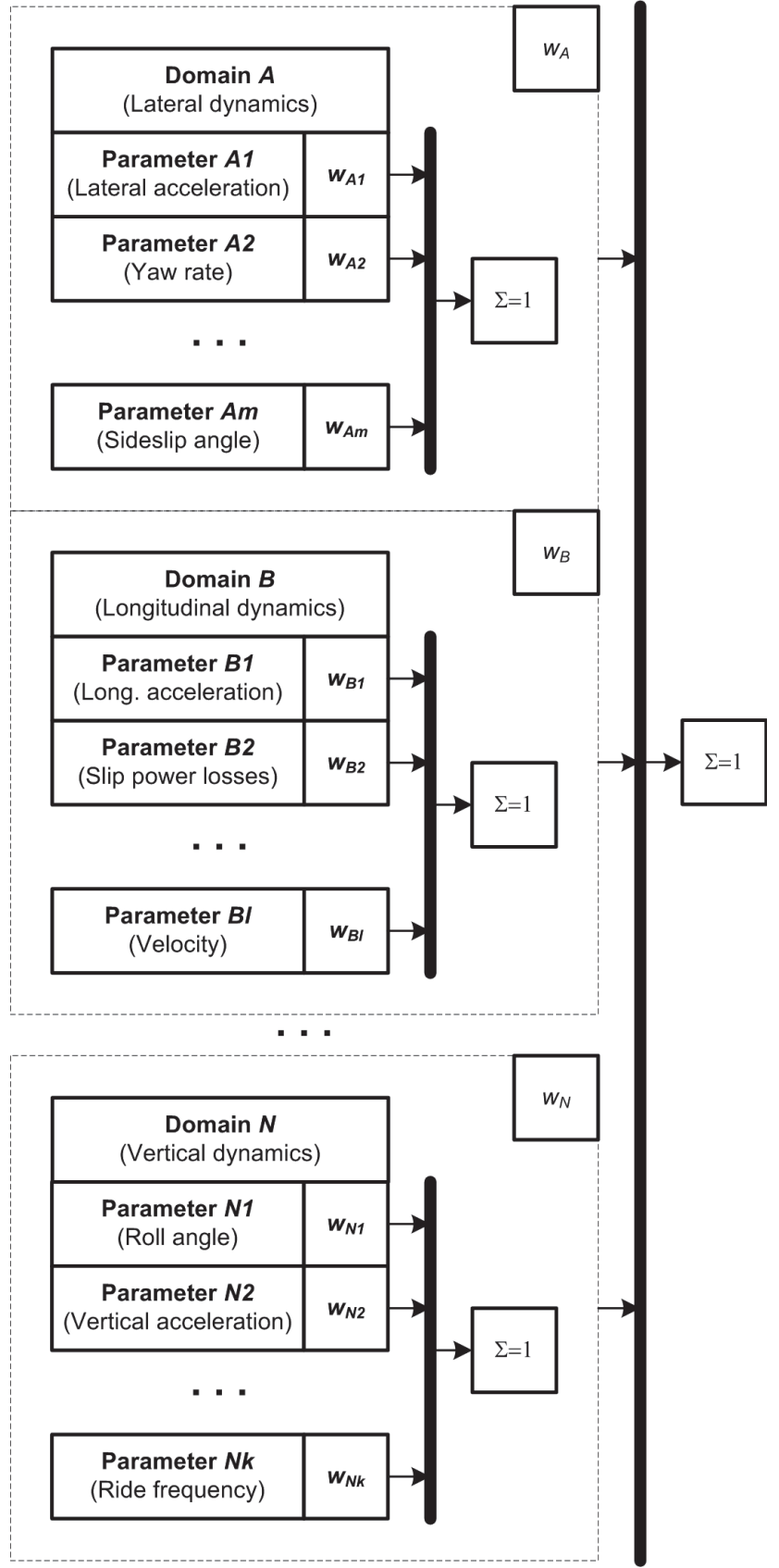

Fig. 1. Computing of cost functions

The composition of weighting factors depends on the type of maneuver that will be explained in next section.

4) Calculation of the individual cost functions:

$E_{A}=\sum_{i=1}^{m} A_{i} \cdot w_{A i} ; E_{B}=\sum_{i=1}^{l} B_{i} \cdot w_{B i} ; \ldots ;$
$E_{N}=\sum_{i=1}^{k} N_{i} \cdot w_{N i}$ 
In accordance with previous allowances, values of individual cost functions belong to the numerical interval $[0$; $1]$.

5) To deduce the complex cost function covering several domains of vehicle dynamics, the weighting factors are designated to individual cost functions (see factors $w_{A}, w_{B}$ and $w_{N}$ on Fig. 1). The sum of the domain-based weighting factors has to be equal to 1 .

6) Computation of the complex (global) cost:

$$
E_{\text {global }}=E_{A} \cdot w_{A}+E_{B} \cdot w_{B}+\ldots E_{N} \cdot w_{N}
$$

The values of the resulting function $E_{\text {global }}$ will be within the range $[0 ; 1]$.

Next section of the paper introduces an example of the composition of cost functions for domains of lateral, longitudinal and vertical dynamics.

\section{EXAMPLE OF COMPOSITION OF COST FUNCTIONS}

\section{A. Cost function of lateral dynamics}

The proposed individual cost function for the lateral dynamics $E_{\text {lat }}$ uses the parameters of the lateral acceleration $a_{y}$, the sideslip angle $\beta$, and the yaw rate $d \psi / d t$. These parameters can be measured by conventional vehicle sensors or derived from vehicle models realized in on-board automotive control systems like ESC. The formulation of the function $E_{\text {lat }}$ is as follows:

$E_{\text {lat }}=w_{a y} \cdot f\left(\Delta a_{y}\right)+w_{\beta} \cdot f(\Delta \beta)+w_{\psi} \cdot f(\Delta \dot{\psi})$,

where $w_{i}$ are the weighting factors, and

$$
\begin{aligned}
& \Delta a_{y}=\left|a_{y}^{r e f}-a_{y}^{a}\right|, \Delta \beta=\left|\beta^{r e f}-\beta^{a}\right|, \\
& \Delta \dot{\psi}=\left|\dot{\psi}^{r e f}-\dot{\psi}^{a}\right|
\end{aligned}
$$

The function $f$ in Eq. (5) as well as in the subsequent formulations is defined by Eq. (1).

The reference lateral acceleration is calculated as

$$
a_{y}^{r e f}=\left\{\begin{array}{l}
a_{y}^{*}, \quad a_{y}^{*} \leq a_{y \max } \\
a_{y \max }, \quad \text { otherwise }
\end{array} .\right.
$$

The maximal lateral acceleration $a_{y}{ }^{*}$ can be defined from the tire friction ellipse as

$$
a_{y \max }=9.81 \cdot \mu_{y \max },
$$

where $\mu_{y \max }$ is the maximal lateral friction coefficient at given level of longitudinal acceleration / deceleration. The parameter $a_{y}{ }^{*}$ in Eq. (7) identifies the reference lateral acceleration that does not exceed the friction limits and can be derived from the look-up-table (LUT) given for the vehicle as a family of "Steering wheel angle - Lateral acceleration"functions composed for different longitudinal accelerations $a_{x}$, Fig. 2. The procedure of the LUT composition is introduced in details in [7].

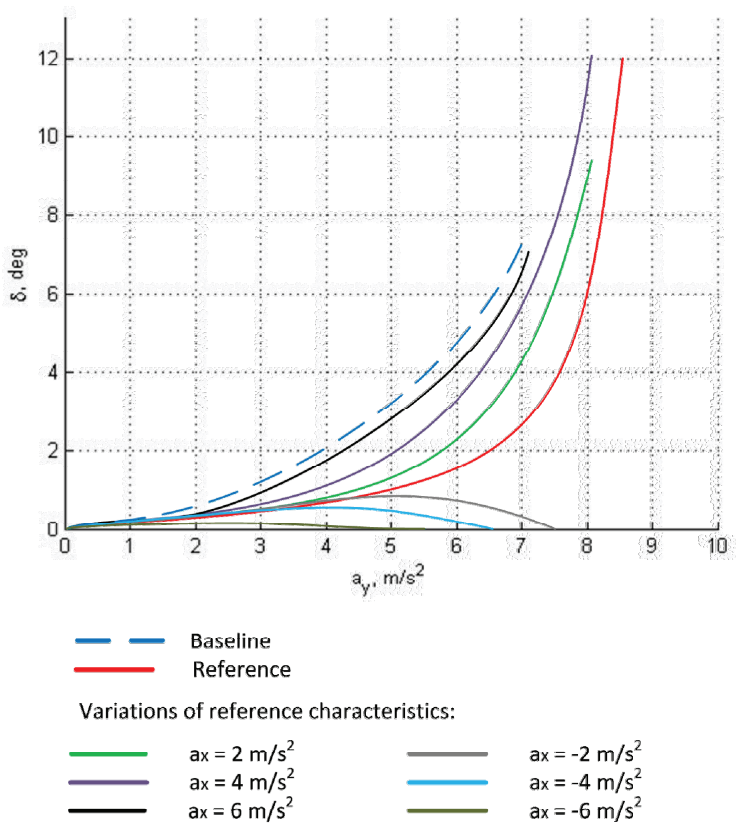

Fig. 2. Example of definition reference data for lateral acceleration

The calculation of reference yaw rate $(d \psi / d t)^{r e f}$ follows the condition:

$$
\dot{\psi}^{r e f}= \begin{cases}\dot{\psi}^{*}, \dot{\psi}^{*} \leq \dot{\psi}_{\max } \\ \dot{\psi}_{\max }, & \text { otherwise }\end{cases}
$$

By analogy with $a_{y}$, the parameter $(d \psi / d t)^{*}$ in Eq. (9) can be derived from look-up tables given for the vehicle as a family of "Steering wheel angle - Yaw rate"-dependencies composed for different longitudinal accelerations. The maximum value of yaw rate can be in addition controlled as [8]

$$
\dot{\psi}_{\max }=\frac{a_{y \max }-\dot{v}_{x} \cdot \sin \beta_{r e f}}{v_{x} \cdot \cos \beta_{r e f}} .
$$

The reference sideslip angle is calculated in accordance with the following conditions 


$$
\beta^{r e f}=\left\{\begin{array}{l}
\beta^{*}, v \leq v_{s s} \\
\beta_{\max \_} s s, \quad \text { otherwise }
\end{array}\right.
$$

The value of sideslip angle for steady-state conditions can be chosen as:

$$
\beta^{*}=\beta_{\text {max }_{-} s s} \frac{v^{2}}{v_{s s}^{2}},
$$

where $v$ is the actual absolute vehicle velocity, $\beta_{\text {max } s s}$ and $v_{s s}$ are correspondingly the maximal sideslip angle and absolute vehicle velocity given for the point where the influence of velocity on yaw rate becomes negligible (for reference, $\beta_{\text {max_ss }}=3^{\circ}$ and $v_{s s}=40 \mathrm{~km} / \mathrm{h}$ were chosen in the presented study based on statistical data).

The actual vehicle sideslip angle $\beta^{a}$ can be computed as follows [3]:

$$
\beta^{a}=\tan ^{-1}\left(v_{y} / v_{x}\right)
$$

where $v_{x}$ is the actual longitudinal velocity, $v_{y}$ is the actual lateral velocity.

It should be mentioned that the comparison of $\beta^{a}$ and $\beta^{\text {ref }}$ takes place only in the case $\beta^{a}>\beta^{\text {ref }}$.

\section{B. Cost function of longitudinal dynamics}

As in the case of the lateral dynamics, the individual cost functions for the assessment of the longitudinal dynamics is based on the parameters that can be measured by conventional on-board sensors or derived from relevant sensor information. Two following parameters are proposed in this context: longitudinal acceleration (deceleration) $a_{x}$, and slip power losses of the vehicle $P_{s \Sigma}$. In such a case the basic formulation of the cost function can be proposed as

$$
E_{\text {long }}=w_{a x} \cdot f\left(\Delta a_{x}\right)+w_{P} \cdot f\left(\Delta P_{s \Sigma}\right)
$$

where $w_{i}$ are the weighting factors and

$$
\begin{gathered}
\Delta a_{x}=\left|a_{x}^{r e f}-a_{x}^{a}\right| \\
\Delta P_{s \Sigma}=\left|P_{s \Sigma}^{r e f}-P_{s \Sigma}^{a}\right| .
\end{gathered}
$$

The longitudinal acceleration is calculated as

$$
a_{x}^{r e f}=\left\{\begin{array}{l}
a_{x}^{*}, \quad a_{x}^{*} \leq a_{x \max } \\
a_{x \max }, \quad \text { otherwise }
\end{array} .\right.
$$

The maximal longitudinal acceleration can be defined from the tire friction ellipse as

$$
a_{x \max }=9,81 \cdot \mu_{x \max }
$$

where $\mu_{x \max }$ is the maximal longitudinal friction coefficient at given level of lateral acceleration / deceleration.

The parameter $a_{x}{ }^{*}$ in Eq. (17) identifies the reference longitudinal acceleration that does not exceed the friction limits.

The value of actual longitudinal acceleration $a_{x}{ }^{a}$ is obtained either (i) with conventional accelerometer being a component of vehicle dynamics control system or (ii) from the vehicle simulator.

The slip power losses of the vehicle can be calculated as:

$$
P_{s \Sigma}=\sum_{i=1}^{n} T_{w i} \cdot \omega_{i} \cdot s_{i}
$$

where $n$ is the number of drive wheels, $T_{w}$ is the wheel torque, $\omega$ is the rotational wheel velocity, $s$ is the wheel slip coefficient.

To calculate the reference slip power losses from Eq. (19), a certain threshold of wheel slip coefficient can be proposed. Based on typical friction-slip-curves, the threshold can correspond to the area of maximal friction coefficient, i.e. $s=0.02 \ldots 0.1$. To calculate the actual slip power losses from Eq. (19), the wheel slip computed from the information of wheel rotational velocity sensors can be used.

\section{Cost function of vertical dynamics}

Within the framework of the presented study, the oneparameter individual cost function is used for the vertical dynamics. This function operates with the roll angle $\theta$.

$$
E_{\text {vert }}=w_{\theta} \cdot f(\Delta \theta),(20)
$$

where the weighting factor $w_{\theta}=1$ in the case under consideration and

$$
\Delta \theta=\left|\theta^{r e f}-\theta^{a}\right|
$$

The reference roll angle can be chosen as the threshold $\theta^{\text {ef }}=1$. As for the sideslip angle, the comparison of $\theta^{t}$ and $\theta^{\text {ref }}$ takes place only in the case $\theta^{a}>\theta^{\text {eef }}$.

\section{Global cost function}

The global cost function in accordance with Eq. (4) can be computed as follows:

$$
E_{\text {global }}=E_{\text {long }} \cdot w_{\text {long }}+E_{\text {lat }} \cdot w_{\text {lat }}+E_{\text {vert }} \cdot w_{\text {vert }} .
$$




\section{E. Choice of weighting factors}

The choice of weighting factors can be done using metaheuristic analysis for different maneuvers and driving situations. The procedure of the definition of the weighting factors is not described in detail in the presented study. However Table I gives examples of composition of weighting factors that were used in the calculations discussed in subsequent sections of the paper.

TABLE I. EXAMPLES F WEIGHTING FACTORS

\begin{tabular}{|c|l|c|}
\hline $\begin{array}{c}\text { Weighing } \\
\text { factors }\end{array}$ & \multicolumn{1}{|c|}{ Maneuver } & $\begin{array}{c}\text { Numerical } \\
\text { value }\end{array}$ \\
\hline \multirow{2}{*}{$w_{a y}$} & Steady-state (e.g. constant circle) & 0.6 \\
\cline { 2 - 3 } & Transient (e.g. avoidance) & 0.2 \\
\hline \multirow{2}{*}{$w_{\beta}$} & Steady-state (e.g. constant circle) & 0.05 \\
\cline { 2 - 3 } & Transient (e.g. avoidance) & 0.3 \\
\hline \multirow{2}{*}{$w_{\psi}$} & Steady-state (e.g. constant circle) & 0.35 \\
\cline { 2 - 3 } & Transient (e.g. avoidance) & 0.5 \\
\hline \multirow{2}{*}{$w_{a x}$} & Steady-state (e.g. constant circle) & 0.6 \\
\cline { 2 - 3 } & Transient (e.g. avoidance) & 0.4 \\
\hline \multirow{2}{*}{$w_{P}$} & Steady-state (e.g. constant circle) & 0.4 \\
\cline { 2 - 3 } & Transient (e.g. avoidance) & 0.6 \\
\hline \multirow{2}{*}{$w_{\theta}$} & Steady-state (e.g. constant circle) & 1.0 \\
\cline { 2 - 3 } & Transient (e.g. avoidance) & 1.0 \\
\hline \multirow{2}{*}{$w_{\text {lat }}$} & Steady-state (e.g. constant circle) & 0.5 \\
\cline { 2 - 3 } & Transient (e.g. avoidance) & 0.5 \\
\hline \multirow{2}{*}{$w_{\text {long }}$} & Steady-state (e.g. constant circle) & 0.2 \\
\cline { 2 - 3 } & Transient (e.g. avoidance) & 0.15 \\
\hline \multirow{2}{*}{$w_{\text {vert }}$} & Steady-state (e.g. constant circle) & 0.3 \\
\cline { 2 - 3 } & Transient (e.g. avoidance) & 0.35 \\
\hline
\end{tabular}

IV. CAse Study: Assessment of TRAnSIENT ManeUEVRS OF THE VEHICLE EQUIPPED WITH ESC SYSTEM

This section illustrates the implementation of the cost functions to assess the vehicle dynamics during maneuvers.

\section{A. Test vehicle and simulator}

The Skoda Yeti vehicle has been used in the study as the test object. The main technical data of the vehicle are given in Table II. The test vehicle is equipped with ESP system. The system ESP comprises several subsystems: ABS (Anti-lock braking system), MSR (Engine Drag Torque Control), ASR (Traction slip control), EDS (Electronic differential lock), and own stability control system ESC.

The experimental results have confirmed that the operation of ESP system improves understeer characteristics of the car, Fig. 3. For numerical assessment of this and other effects, the described methodology was applied.

To compute and analyze the cost functions, a number of maneuvers were performed using the instrumented vehicle, Fig. 4, and then investigated with the full vehicle simulator created in IPG CarMaker software environment.

Fig. 5 presents the results of the validation of the vehicle simulator by the example of maneuver "Track keeping - circle with radius $50 \mathrm{~m}$ ". Next sub-section presents the calculation of the cost functions for more complex, transient maneuvers.
TABLE II. VEHICLE TECHNICAL DATA

\begin{tabular}{|l|l|}
\hline \multicolumn{1}{|c|}{ Parameter } & \multicolumn{1}{|c|}{ Value / Description } \\
\hline Total weight & $2080 \mathrm{~kg}$ \\
\hline Maximum speed & $201 \mathrm{kph}$ \\
\hline Acceleration 0-100 kph & $8,4 \mathrm{~s}$ \\
\hline Front axle & $\begin{array}{l}\text { McPherson suspension with lower triangular } \\
\text { links and transverse torsion stabiliser }\end{array}$ \\
\hline Rear axle & $\begin{array}{l}\text { Multi-element suspension with a } \\
\text { longitudinal and transverse links and } \\
\text { transverse torsion stabiliser }\end{array}$ \\
\hline Steering & $\begin{array}{l}\text { Direct rack-and-pinion steering with } \\
\text { electromechanical power steering }\end{array}$ \\
\hline Tyres & $215 / 60 \mathrm{R} 16$ \\
\hline Dimensions & $4223 \mathrm{~mm}$ x 1793 mm x 1691 mm \\
\hline Wheelbase & $2578 \mathrm{~mm}$ \\
\hline Outer turning circle diameter & $10,32 \mathrm{~m}$ \\
\hline
\end{tabular}

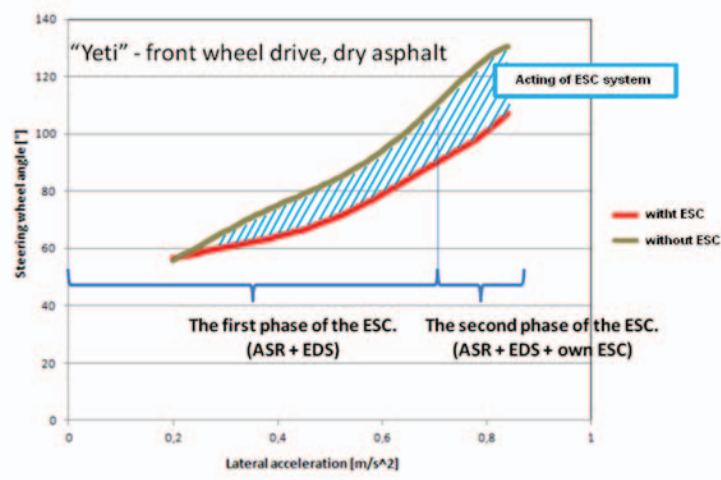

Fig. 3. Influence of ESC opeartion on understeer characteristics
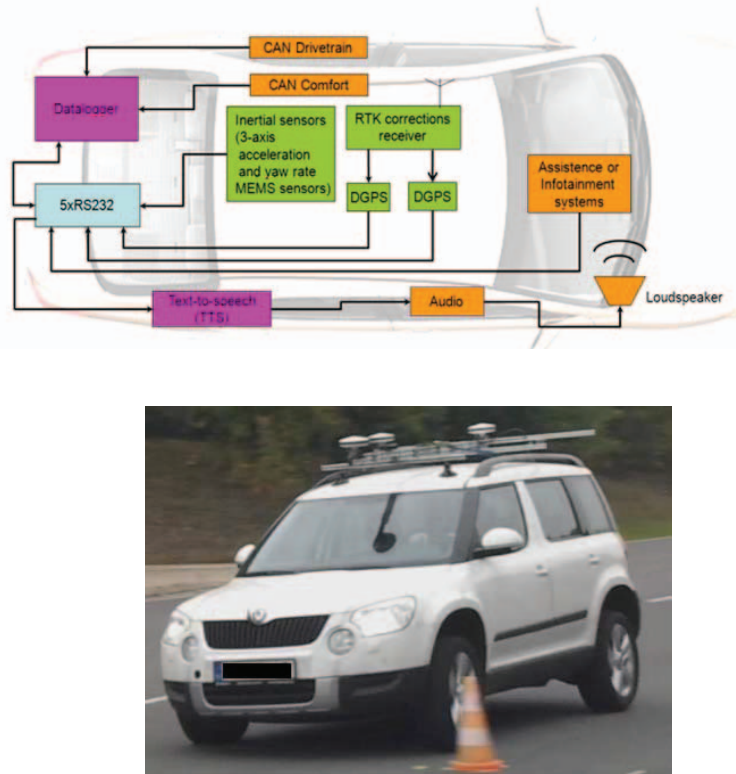

Fig. 4. Test Vehicle 


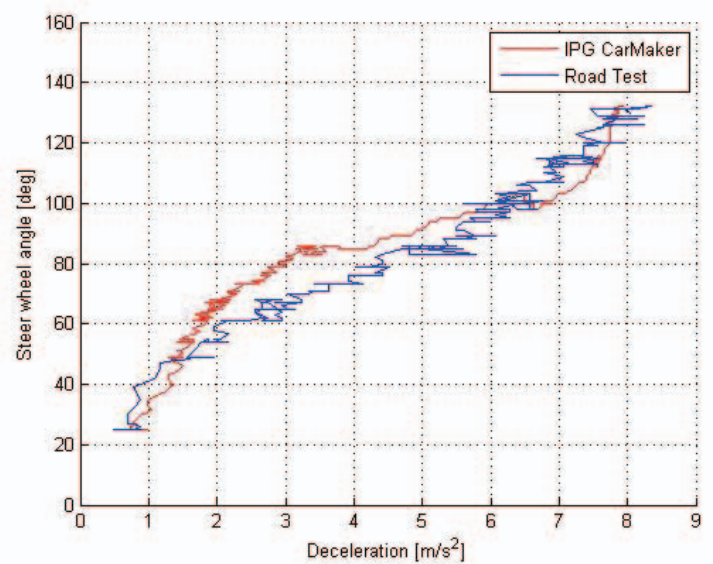

Fig. 5. Validation of vehicle simulator on IPG CarMaker

\section{B. Tests on the vehicle simulator}

Two maneuvers were chosen for the calculation of the cost functions for the vehicle simulator: slalom (standard ISO 7401) and avoidance (standard ISO 17512). Each maneuver has been simulated with three operation modes: driving without ESP, driving with conventional ESP configuration, driving with modified ESP configuration. The modified ESP configuration has been developed after the analysis of the cost functions obtained for the conventional ESP configuration with the aim to minimize the global cost function $E_{\text {global }}$. For this purpose, several parameters of ESP controller can be varied. These are the gain of regulator and the threshold, reaching which ESP systems starts operating. Fig. 6-9 present the main test results. The cost functions were computed in accordance with equations and weighting factors presented in section III. The reference data have been used as for the vehicle without ESP operation. The analysis of results allows to draw following conclusions:

1) The complexity of maneuver can be properly evaluated with the proposed methodology. For instance, the avoidance as a less transient maneuver as compared with the slalom has received lower maximum values of the individual cost function of lateral dynamics ( 0.055 vs. 0.095 with conventional ESP) and the complex cost function ( 0.11 vs. 0.15$)$.

2) The use of the individual and complex cost functions gives the basis for the tuning of ESP control algorithms to improve the controlled vehicle operation in terms of stability and performance. In particular, the modified ESP has revealed during the slalom maneuver the improved stability performance (maximum of the individual cost functions was reduced on 0.035 points) and better overall dynamics (maximum of the complex cost functions was reduced on 0.025 points).

Additional numerical analysis of the test results for the vehicle simulator will be addressed in the extended journal version of this paper.

\section{CONCLUSIONS}

The methodology introduced in the paper has described the procedures of the calculation of the cost functions for (i) individual assessment of lateral, longitudinal, and vertical vehicle dynamics, and (ii) complex assessment of global dynamic behavior of the vehicle. The cost functions are based on the comparison of actual and reference values of lateral and longitudinal acceleration, yaw rate, sideslip angle, wheel slip losses, and roll angle.

The calculation of cost functions was illustrated with the case study for modeling of transient maneuvers (slalom and avoidance) on the vehicle simulator. At that, the vehicle simulator was validated on the basis of experiments on the real vehicle.

The developed method for the computation of the cost functions can find applications not only for the assessment of vehicle dynamics but also for optimization of algorithms of vehicle control systems.

\section{REFERENCES}

[1] J. Ellis, Vehicle handling dynamics, Professional Engineering Publishing, 1994

[2] R. Rajamani, Vehicle dynamics and control, Springer, 2006.

[3] J.Y. Wong, Theory of Ground Vehicles, John Wiley \& Sons, Inc., New York, 2001

[4] W.F. Milliken and D.L. Milliken, Chassis Design: Principles and Analysis, SAE International, 2002.

[5] H.S. Radt and D.A. Glemming, "Normalization of Tire Force and Moment Data", Tire Science and Technology, Vol. 21, No. 2, pp. 91119, 1993.

[6] H. Peng and M. Tomizuka, "Vehicle Lateral Control for Highway Automation", Proc. of American Control Conference, pp. 788-794, 1990.

[7] V. Ivanov, K. Augsburg, D. Savitski, J. Plihal, P. Nedoma and J. Machan, „Advanced Cost Functions for Evaluation of Lateral Vehicle Dynamics",Lecture Notes in Electrical Engineering, Vol. 198, pp. 425440, Springer, 2013.

U. Kiencke and L. Nielsen, Automotive Control Systems, Springer-Verlag, Berlin-Heidelberg, 2005. 

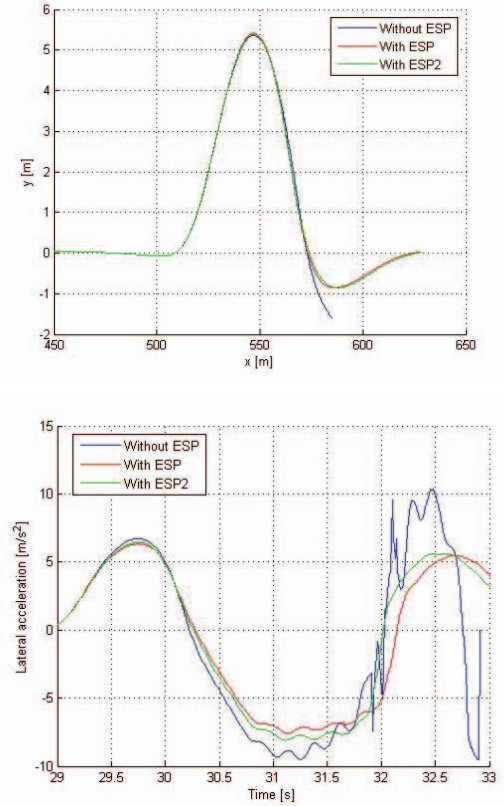

Fig. 6. Dynamic variables for avoidance maneuver
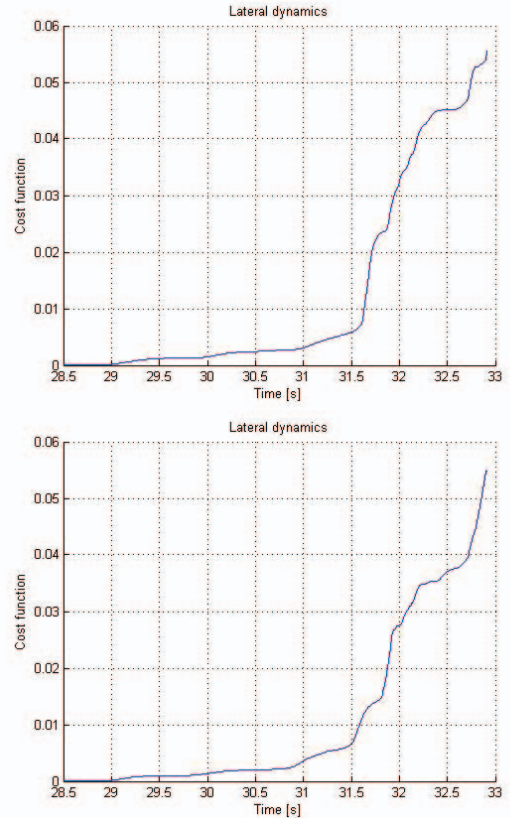
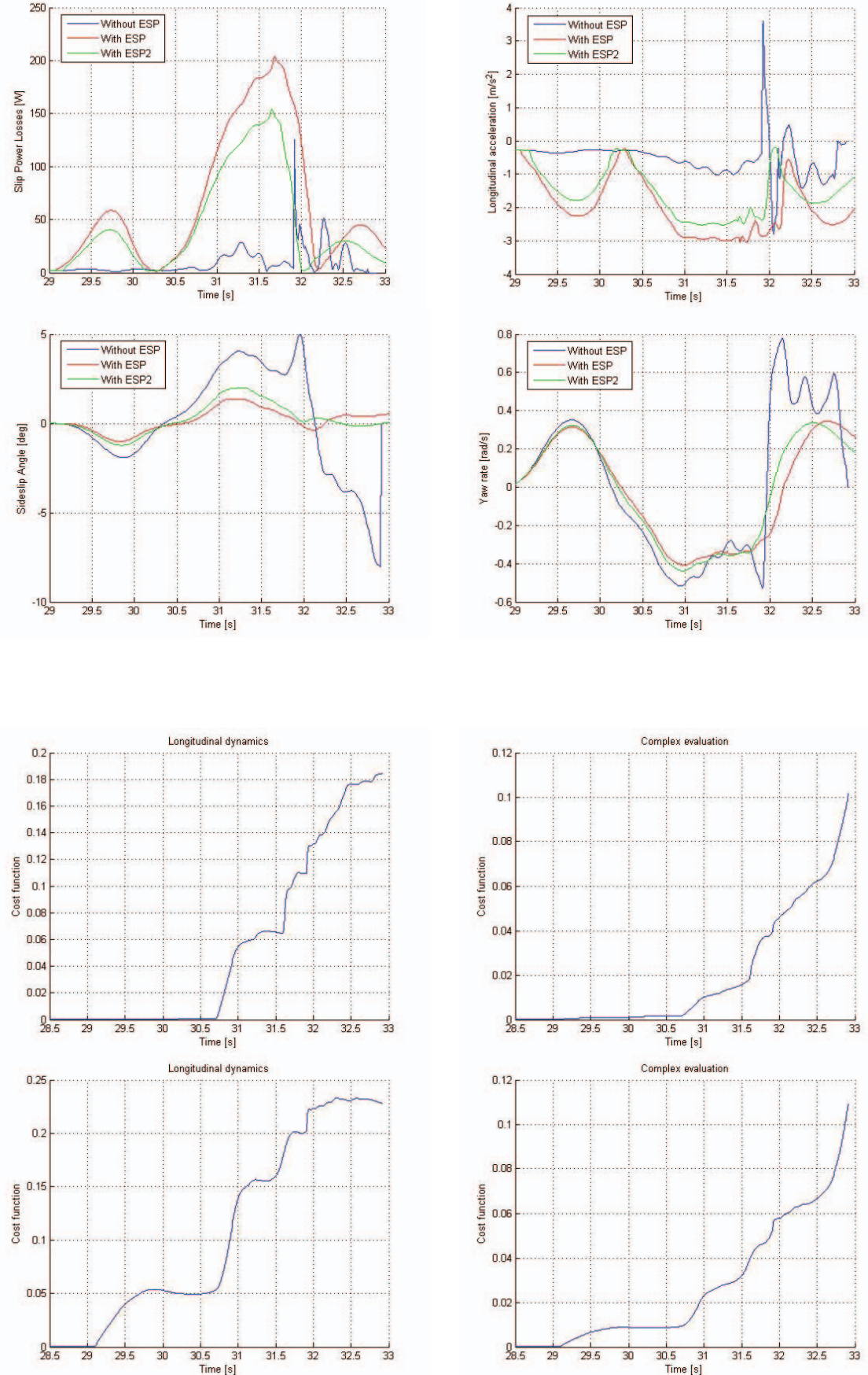

Fig. 7. Cost functions for avoidance maneuver (upper row - conventional ESP, lower row - modified ESP) 

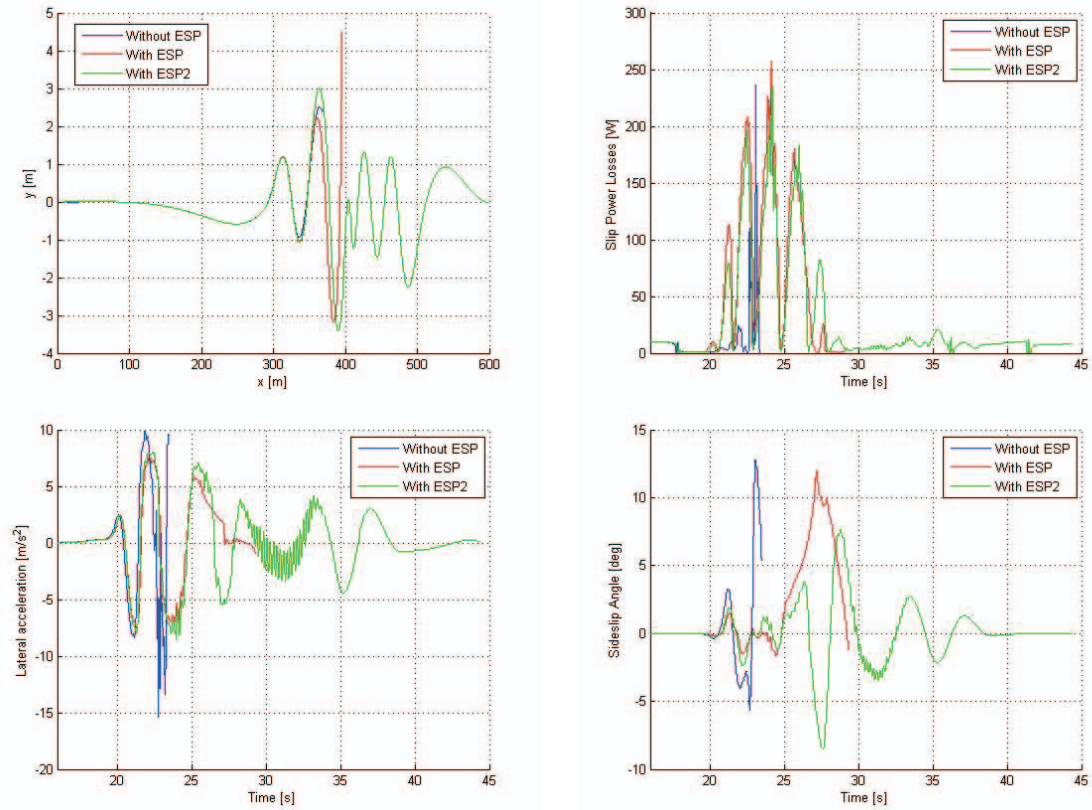

Fig. 8. Dynamic variables for slalom maneuver
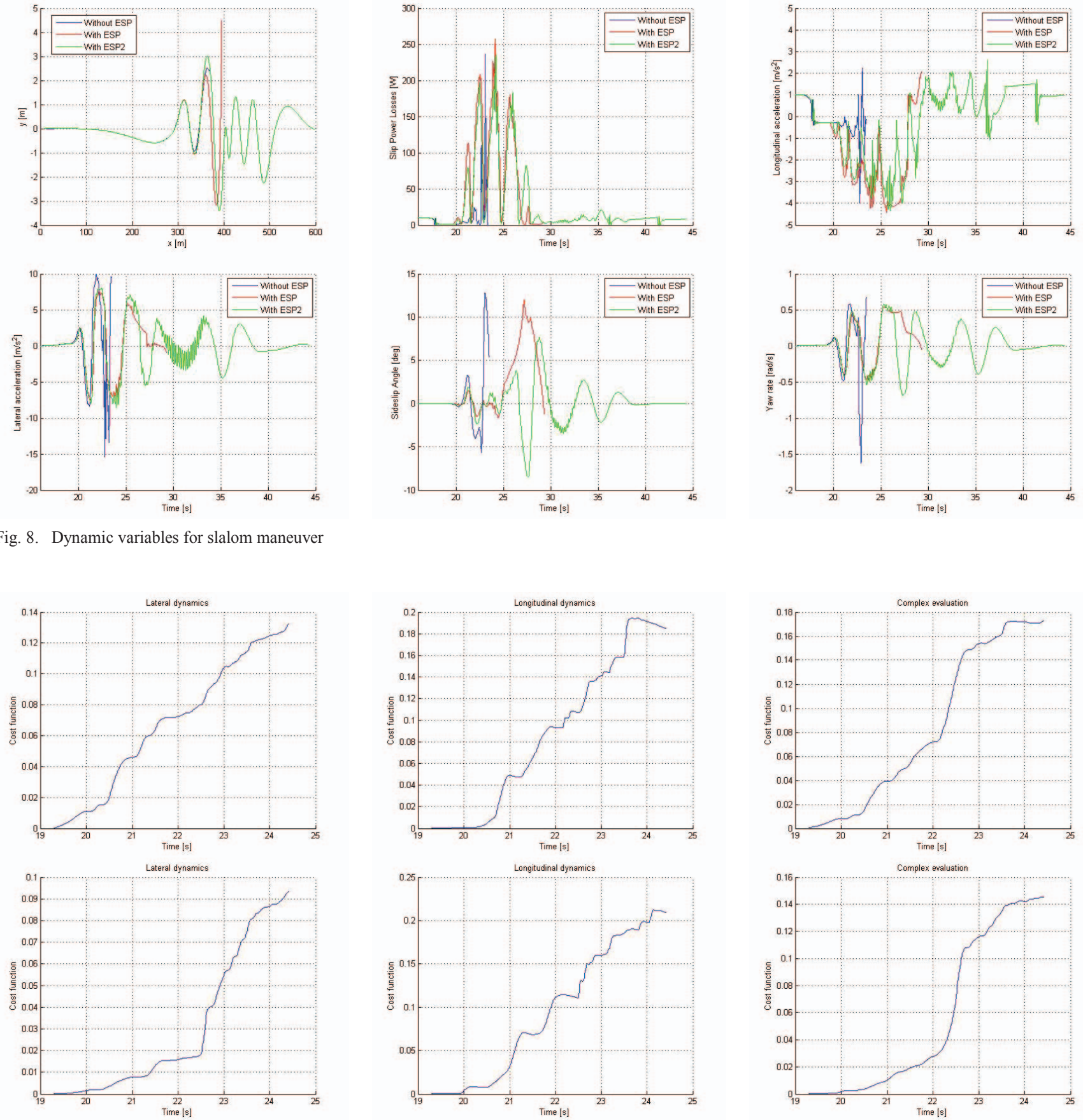

Fig. 9. Cost functions for slalom maneuver (upper row - conventional ESP, lower row - modified ESP) 